\title{
Retenção do dimetoato e sua relação com pH e teores de argila e matéria orgânica nos sedimentos da zona não- saturada de uma microbacia no nordeste paraense
}

\author{
Lilianne Maia LIMA¹, Eliene Lopes de SOUZA², Ricardo de Oliveira FIGUEIREDO ${ }^{3}$
}

\begin{abstract}
RESUMO
$\mathrm{Na}$ agricultura familiar na Amazônia oriental, em particular no nordeste do Pará, são comuns os cultivos semi-perenes com pesada aplicação de agrotóxicos. Em virtude da ampla utilização desses produtos, principalmente o dimetoato, na microbacia hidrográfica do igarapé Cumaru, município de Igarapé-Açu (PA), foi avaliada a retenção dessa substância em amostras da zona não-saturada em laboratório, verificando-se também a influência do $\mathrm{pH}$ e dos teores de argila e de matéria orgânica nesse processo. Entre os diversos agrotóxicos utilizados na área, o dimetoato foi selecionado por apresentar maior potencial de lixiviação, segundo o índice GUS (Groundwater Ubiquity Score). Para a quantificação da retenção do dimetoato nos sedimentos da zona não-saturada foi realizado um experimento de sorção. Este último mostrou que, em termos percentuais, a sorção do dimetoato variou de $2.5 \%$ a $36.2 \%$ (concentração inicial $20 \mathrm{mg}^{-1}$ ) e de $6.20 \%$ a $31.0 \%$ (concentração inicial $10 \mathrm{mg}^{-1}{ }^{-1}$ ). Esses dados comprovam o elevado potencial de contaminação da água subterrânea por essa substância, devido, principalmente, à sua mobilidade e baixa retenção. Devido ao caráter hidrofóbico do dimetoato, que aumenta a sua afinidade com a matéria orgânica, a quantidade sorvida dessa substância se mostrou diretamente proporcional à de matéria orgânica presente nos sedimentos. $\mathrm{O}$ pH exerce efeito contrário a este, ou seja, quanto mais elevado o seu valor, menor é a quantidade de dimetoato sorvida. Em relação à variação do teor e ao tipo de argila, foi observado que esses fatores não influenciam na retenção do dimetoato, sendo esse resultado atribuído ao comportamento não iônico desse agrotóxico.
\end{abstract}

PALAVRAS-CHAVE

Agrotóxico, água subterrânea, agricultura familiar, lixiviação

\section{Dimethoate retention and its relation to $p H$, clay and organic mater contents by sediments in non saturated zone of a small catchment of the northeast Pará.}

\begin{abstract}
On smallholder farms of eastern Amazonia, in particular the northeast of the Pará state, heavy by pesticide applications at industrial halfperennial crops are very common. Due to their large use, mainly the dimethoate, in the catchment of the Cumaru stream, Igarapé-Açu county, we evaluated the retention process of this compound in samples of the non saturated zone, as well as verified the effects of soil $\mathrm{pH}$ and clay and organic mater contents in this process. Among the main pesticides the farmers use in this region, dimethoate was selected for this study, due to its larger leaching potential according to the GUS (Groundwater Ubiquity Score) index. A sorption experiment was carried out to evaluate the retention of the dimethoate in sediments in the non saturated zone. The experiment showed that the sorption of the dimethoate varied from $2.5 \%$ to $36.2 \%$ (initial concentration of $20 \mathrm{mg} . \mathrm{t}^{-1}$ ) and from $6.2 \%$ to $31.0 \%$ (initial concentration of $10 \mathrm{mg} . \mathrm{l}^{-1}$ ). These data demonstrate a high contamination potential of the groundwater by this compound, mainly due to its high mobility and low retention capacity. The dimethoate sorption rates were positively related to organic matter content in the sediments. On the other hand, values have an inverse relationship with the sorption rates of dimethoate, while clay content did not produce any effect on its retention.
\end{abstract}

\section{KEY WORDS}

Pesticide, groundwater, smallholder farm, leaching.

\footnotetext{
${ }^{1}$ Mestre em Hidrogeologia, Embrapa Amazônia Oriental (Projeto TIPITAMBA), Tv. Enéas Pinheiro s/n, Bairro do Marco, CEP 66095-100, Belém-Pará. e-mail: lilianne_maia@yahoo.com.br 2Dra em Geologia e Geoquímica, Universidade Federal do Pará, Centro de Geociências, Rua Augusto Corrêa s/n, Bairro do Guamá, CEP 66075-110, Belém-Pará. e-mail: eliene@ufpa.br ${ }^{3}$ Dr. em Ciências Ambientais, Embrapa Amazônia Oriental, Tv. Enéas Pinheiro s/n, Bairro do Marco, CEP 66095-100, Belém-Pará. e-mail: ricardo@cpatu.embrapa.br.
} 


\section{INTRODUÇÃo}

A utilização de agrotóxicos representa um risco ambiental de grande magnitude e com elevados custos econômicos. Além de contaminar o ar, o solo, as águas superficiais e subterrâneas, causam graves danos à saúde, tanto dos trabalhadores que manipulam os produtos químicos como dos consumidores de alimentos contaminados com resíduos de substâncias tóxicas (Poltroniéri, 1997).

A contaminação das águas superficiais e subterrâneas em áreas com aplicação de agrotóxicos ocorre principalmente através dos processos de escoamento e lixiviação, afetados pela sorção, processo que vem recebendo muita atenção de pesquisadores nas últimas décadas. A sorção é influenciada pelas propriedades do agrotóxico e do solo (Silva, 1994; Singh, 2002), destacandose a mineralogia e os teores de argila e de matéria orgânica (Vieira et al., 1999; Lopes et al., 2002; Spark \& Swift, 2002).

Vieira et al. (1999) estudaram a adsorção/desorção do ácido 2,4 diclorofenoxiacético $(2,4 \mathrm{D})$ em solo, na ausência e na presença de matéria orgânica, verificando que os valores do coeficiente de adsorção no solo livre de matéria orgânica foram relativamente baixos, quando comparados com os do solo contendo matéria orgânica. Nesse mesmo estudo foi verificado que o agrotóxico 2,4- D não adsorve muito nas argilas, sendo este resultado atribuído ao fato de que as cargas negativas dos colóides do solo repelem o agrotóxico.

Lopes et al. (2002) estudaram o efeito da matéria orgânica do solo na adsorção de agrotóxicos, neste caso o triadimenol. Os autores concluíram que a afinidade dessa substância pela matriz do solo aumenta com o teor de matéria orgânica.

Ao estudar a sorção do herbicida imazaquim em solos com balanço de carga positiva, Rocha et al. (2002) verificaram que a sorção é influenciada principalmente pelo $\mathrm{pH}$ e pela carga elétrica líquida do solo. O herbicida apresenta baixa sorção ao solo e, tanto em superfície quanto em profundidade, a sorção diminui com a elevação do $\mathrm{pH}$.

Gao et al. (1998) estudaram a sorção de pesticidas nos sedimentos do sul da Alemanha. Os autores notaram que a capacidade de sorção dos sedimentos é diretamente relacionada com o conteúdo de matéria orgânica e o $\mathrm{pH}$. Ao remover a matéria orgânica dos sedimentos, os autores observaram que a capacidade de sorção é reduzida. Além disso, verificaram também que o aumento do $\mathrm{pH}$ causa decréscimo na sorção dos pesticidas.

As propriedades das substâncias também influenciam na sorção. Este fato foi verificado por Nemeth-Konda et al.(2002) ao investigarem a sorção de seis substâncias diferentes. Os autores observaram que a sorção do carbendazin e diazinon é maior que a do imidacloprid, isoproturon e triazina, sugerindo que nos solos estudados a migração dos agrotóxicos carbendazin e diazinon para a água subterrânea seria menor que a dos outros examinados.
Pessoa et al. (2003) realizaram simulaçōes com o objetivo de analisar a movimentação vertical dos herbicidas atrazina, diuron e tebutiuron, bem como a tendência futura de risco de contaminação da água subterrânea. De acordo com esse estudo, o herbicida tebutiuron alcançou maiores profundidades em todos os solos simulados, quando comparado às outras substâncias. Nenhum dos herbicidas alcançou profundidades que atingissem a zona saturada, situada a partir de 40 metros.

$\mathrm{Na}$ bacia do igarapé Cumaru, localizada no município de Igarapé-Açu (PA), os agrotóxicos são utilizados com bastante frequiência, principalmente no cultivo do maracujá. Em torno de $46 \%$ dos produtores efetuam 4 aplicaçôes mensais, 29\% efetuam 8 aplicações mensais, $11 \%$ duas aplicaçōes mensais, $11 \%$ uma vez ao ano e $3 \%$ duas vezes ao ano (Amaral, 2001). De acordo com esses números, constata-se que, em sua maioria, os agricultores utilizam os agrotóxicos fora do recomendado pelo Ministério da agricultura apud Amaral (2001), segundo o qual as aplicações devem ser espaçadas no mínimo por 15 dias, independentemente da incidência das pragas e do tipo de produto.

Em virtude da ampla utilização de agrotóxicos na bacia hidrográfica do igarapé Cumaru, principalmente o dimetoato, buscou-se avaliar, em laboratório a retenção dessa substância em amostras da zona não-saturada, bem como os fatores que a influenciam. Entre os diversos agrotóxicos utilizados nessa bacia selecionou-se o dimetoato [0,0-dimetil-S-metilcarbamoilmetil fosforoditioato] para avaliação, por se tratar de uma substância do grupo dos organofosforados, muito perigosa ao meio ambiente, altamente móvel, apresentando alto potencial de deslocamento no solo, podendo atingir as águas subterrâneas (The Pesticide Manual, 2001).

\section{ÁREA DE ESTUDO}

A área em estudo situa-se na bacia hidrográfica do igarapé Cumaru, com 54.1 ha, a $12 \mathrm{~km}$ da sede do município de IgarapéAçu , na região nordeste do Estado do Pará, distante $110 \mathrm{~km}$ de Belém, sua capital (Figura 1). Em Igarapé-Açu, como acontece na Amazônia, a variação climática está associada com a distribuição das chuvas. Entre os anos de 1994 a 1998, o total de chuvas anual variou entre 2.300 e $2.800 \mathrm{~mm}$ (Bastos \& Pacheco, 1999). No entanto, as chuvas não se distribuem homogeneamente durante $o$ ano, ocorrendo com maior freqüência entre março e abril e menor entre setembro e outubro. De acordo com Kanashiro \& Denich (1998), a temperatura média anual de Igarapé-Açu é de $26.6^{\circ} \mathrm{C}$, variando entre $25.6^{\circ} \mathrm{C}$ (março) e $27.5^{\circ} \mathrm{C}$ (novembro). A temperatura máxima flutua entre $30^{\circ} \mathrm{C}$ (abril) e $33.5^{\circ} \mathrm{C}$ (novembro), enquanto que a mínima varia entre $20.2^{\circ} \mathrm{C}$ (março) e $21.9^{\circ} \mathrm{C}$ (dezembro).

Os solos da área são do tipo Argissolo Amarelo Distrófico, de textura arenosa/média, ácidos, profundos e bem drenados. 
$\mathrm{Na}$ área da bacia ocorrem sedimentos inconsolidados, arenosiltosos e areno-argilosos. Esses sedimentos apresentam coloração creme, amarela e amarelo- alaranjada, são pobremente selecionados, e contêm algumas vezes grãos esparsos de quartzo. As características litológicas desses sedimentos, aliadas à análise da geologia regional, permitem associá-los aos Sedimentos PósBarreiras (Góes \& Truckenbrodt, 1980). Sotopostos a estes, a partir de aproximadamente 12 metros de profundidade, ocorrem sedimentos com grandes variações litológicas, indo de argilas até arenitos grosseiros, do Grupo Barreiras. O contexto geológico da área define duas unidades aqüíferas, denominadas de Aqüífero Barreiras e Aqüífero Quaternário, sendo que o estudo se limitou a este último por ser mais superior e mais vulnerável à contaminação.

O Aquiífero Quaternário é constituído por areias de granulometria fina a média, moderadamente selecionadas. Segundo Leão (2003) apresenta cerca $12 \mathrm{~m}$ de espessura. Nas áreas com cotas topográficas mais elevadas o nível estático encontra-se com $13 \mathrm{~m}$ de profundidade, enquanto que nas mais baixas não ultrapassa 3 metros. A transmissividade do aqüífero é de $347 \mathrm{~m}^{2}$.dia e a porosidade efetiva de 0,25 (Leão, 2003).

\section{MATERIAL E MÉTODOS}

\section{CARACTERIZAÇÃO DA ZONA NÃO-SATURADA}

Foram realizadas três perfuraçôes a trado manual a fim de conhecer melhor a geologia da área e coletar amostras da zona não-saturada, destinadas às análises laboratoriais posteriores. Essas análises tiveram por objetivo determinar os teores de argila e matéria orgânica, pH e composição mineralógica, características estas que podem influenciar no processo de sorção. As amostras foram coletadas quando se identificou mudança na litologia. Posteriormente foram homogeneizadas, quarteadas e acondicionadas em sacos plásticos devidamente identificados. A determinação das frações areia, silte e argila foram realizadas através da combinação dos métodos peneiramento e decantação (Suguio,1973). A análise mineralógica foi realizada através do método de difração de raios-X, em laboratório do Centro de Geociências-UFPA. O método consiste em prensar em uma lâmina específica $0,5 \mathrm{~g}$ de amostra seca a $50^{\circ} \mathrm{C}$, homogeneizada e triturada. Para a determinação da composição mineralógica utilizou-se um difratômetro PHILPS, modelo PW3710 com monocromador de grafite e tubo de ânodo de cobre.

O procedimento para determinação do $\mathrm{pH}$ do solo em água seguiu a metodologia proposta por Guimarães et al. (1970). Para essa análise foram misturados $100 \mathrm{~g}$ de amostra de sedimento com $100 \mathrm{ml}$ de água. A mistura foi agitada e deixada em repouso por uma hora; após esse tempo foi agitada novamente, determinando-se imediatamente o $\mathrm{pH}$ da suspensão de solo, por meio de um pHmetro modelo HI 9321, no Laboratório de Química da UFPA.
A determinação do teor de matéria orgânica seguiu a metodologia de análise dicromatométrica (Guimarães et al., 1970). O método baseia-se na ação oxidante do dicromato de potássio (K2Cr2O7) em meio ácido, sobre a fração do solo denominada carbono orgânico.

\section{SELEÇÃO DO AGROTÓXICO PARA OS ENSAIOS EM LABORATÓRIO}

A seleção do agrotóxico utilizado nos ensaios foi baseada no índice Groundwater Ubiquity Score (GUS) (Gustafson,1989), aplicado para o dimetoato, deltamentrina e mancozeb, agrotóxicos utilizados na área. O cálculo desse índice considera os valores de meia-vida do composto no solo (DT ${ }_{50}{ }^{-}$) e o coeficiente de adsorção à matéria orgânica (Koc), sendo obtido através da equação:

$$
\text { GUS }=\log \left(\mathrm{DT}_{50} \text { solo- }\right) \times(4-\log (\text { Koc }))
$$

Os parâmetros $\mathrm{DT}_{50}$ - e Koc foram retirados da literatura (The Pesticide Manual, 2001), ressaltando-se que esses valores foram determinados para clima temperado. Uma vez determinado o índice GUS para cada agrotóxico, os mesmos podem ser classificados em três categorias distintas: GUS = 1,8 (não sofre lixiviação), 1,8<GUS $<2,8$ (faixa de transição), GUS $\geq 2,8$ (provável lixiviação).

\section{EXPERIMENTO DE SORÇÃO}

O método empregado na realização do experimento de sorção foi baseado em estudos realizados por Gao et al. (1998) e NemethKonda et al. (2002), sendo modificadas algumas etapas.

Para realização do experimento foram utilizadas amostras coletadas em um perfil da zona não-saturada, cujas características granulométricas, valores de $\mathrm{pH}$, teores de matéria orgânica e de argila se mostraram similares aos de outros perfis da área. As amostras foram secas ao ar e passadas em peneira de 2,0 $\mathrm{mm}$ de malha. Foram coletadas amostras nas profundidades de 0.0-0.30, 0.30-1.00, 1.00-3.00 e 3.00-5.00 m.

As amostras dos sedimentos previamente preparadas foram colocadas em contato com soluçóes de 10 e $20 \mathrm{mg}^{-1}{ }^{-1}$ de dimetoato e pH 5 e 8 , durante 24 horas. Dessa forma, foram obtidas 16 amostras de resíduo, todas analisadas em duplicatas. As concentrações de dimetoato utilizadas no experimento foram obtidas a partir da diluição da solução estoque de $400 \mathrm{mg} \cdot \mathrm{l}^{-1}$. O pH foi ajustado utilizando-se soluções de $\mathrm{HCl}$ e $\mathrm{NaOH}$.

Os valores de concentração inicial são maiores que os utilizados nos trabalhos de Gao et al. (1998) e Nemeth-Konda et al. (2002), nos quais foi utilizada concentração máxima de $5 \mathrm{mg} . \mathrm{l}^{-}$ ${ }^{1}$. Entretanto, uma vez que se desconhecia o comportamento do dimetoato, sabendo-se apenas que esta substância apresenta meia- 
vida menor do que as das utilizadas pelos autores citados acima, optou-se, por medida de segurança, em utilizar concentrações mais elevadas.

Os valores de $\mathrm{pH}$ foram selecionados de acordo com alguns critérios específicos. O primeiro deles foi o fato do dimetoato ter uma solubilidade conhecida em pH ácido (5) e básico (8). Como segundo critério, procurou-se levar em consideração o $\mathrm{pH}$ natural das águas da região, buscando uma aproximação com situações reais.

Em frascos de vidro escuro, para evitar a fotoxidação, foram misturados $100 \mathrm{~g}$ de cada amostra, 1 litro da solução do dimetoato e $0.01 \mathrm{~g}$ de cloreto de cálcio, a fim de manter a força iônica. $\mathrm{O}$ $\mathrm{pH}$ foi ajustado e a mistura foi agitada durante 24 horas em agitador magnético, à temperatura ambiente. Ao final, as amostras foram centrifugadas por 20 minutos, a $3000 \mathrm{rpm}$. O sobrenadante foi recolhido e conservado a $4^{\circ} \mathrm{C}$ para posterior determinação do agrotóxico através de análises cromatográficas.

\section{DETERMINAÇÃO DO PESTICIDA}

A quantificação do dimetoato foi realizada no laboratório BIOAGRI Ambiental Ltda. (SP), em um cromatógrafo a gás HP6890 acoplado a espectrômetro de massas HP5973 e workstation para integração. Empregou-se uma coluna HP5MS (30 $\mathrm{m} \times 0,25 \mathrm{~mm} \times 0,25 \mu \mathrm{m})$, com temperatura da coluna programada para iniciar em $60^{\circ} \mathrm{C}$, com rampa de aquecimento de $20^{\circ} \mathrm{C}$ por minuto, até $300^{\circ} \mathrm{C}$. A temperatura do injetor foi de $250^{\circ} \mathrm{C}$ e o volume da injeção de $1 \mu \mathrm{l}$, com o fluxo do gás hélio na vazão de $1 \mathrm{ml}^{-1} \cdot$ min Todas as injeções foram efetuadas no modo splitless.

A quantificação do dimetoato sorvido nos sedimentos foi determinada utilizando a seguinte equação (Lopes et al., 2002):

$$
\mathrm{x}=\mathrm{v} / \mathrm{m}\left(\mathrm{C}_{\mathrm{p}}-\mathrm{C}_{\mathrm{e}}\right)
$$

\section{Onde:}

$\mathrm{x}=$ quantidade do dimetoato sorvido por grama do solo (mg.lg$\left.{ }^{1}\right)$

v= volume da solução de dimetoato adicionada (l)

$\mathrm{m}=$ massa do sedimento $(\mathrm{g})$

$\mathrm{C}_{\mathrm{p}}=$ concentração do dimetoato na solução padrão adicionada (mg. ${ }^{-1}$ )

$\mathrm{C}_{\mathrm{e}}=$ concentração do dimetoato na solução em equilíbrio com o solo (mg. $\left.{ }^{-1}\right)$

\section{RESULTADOS E DISCUSSÃO}

\section{CARACTERÍSTICAS DA ZONA NÃO-SATURADA}

As principais características dos sedimentos da zona nãosaturada estão apresentadas na Tabela 1. Esta zona caracteriza-se por apresentar a fração areia predominante em todo o perfil, principalmente nos horizontes superiores e nas proximidades $\mathrm{da}$ superfície freática. A fração areia foi classificada através do software ANASED 5.0 como areia média, moderadamente selecionada.

Verifica-se em todo o perfil que as amostras são constituídas por quartzo e caulinita. Os elevados teores de quartzo estão relacionados com sua elevada estabilidade aos processos intempéricos. Esse mineral se comporta inerte quimicamente nas faixas de $\mathrm{pH}$ e temperatura da região. A dominância da caulinita é condizente com o clima tropical úmido da região Amazônica (Garrels \& Mackenzie apud Goulart, 1982).

Os solos da área em estudo apresentam caráter ácido, com valores de $\mathrm{pH}$ variando de 4.28 a 5.20. No geral, ocorre um ligeiro aumento dos valores de $\mathrm{pH}$ em direção à superfície, devido ao fluxo de água meteórica que lava os horizontes superficiais, removendo os ácidos orgânicos (Goulart, 1982).

Os teores de matéria orgânica nos horizontes investigados são muito baixos, não ultrapassando $3.0 \%$. De modo geral, verifica-se um empobrecimento dos teores de matéria orgânica com a profundidade.

\section{AGROTÓXICO SELECIONADO PARA O ENSAIO DE SORÇÃO}

A Tabela 2 apresenta os valores dos parâmetros utilizados para o cálculo do índice GUS (Groundwater Ubiquity Score) (Gustafson, 1989), bem como os valores do referido índice. Dentre os agrotóxicos mais utilizados na bacia, o dimetoato apresenta maior potencial de lixiviação, sendo classificado entre as faixas de transição e provável lixiviação. Assim, com base no índice GUS, o dimetoato apresenta-se como um provável contaminante das águas subterrâneas. Porém, esta contaminação poderá não ocorrer quando as condições do solo favorecem a sorção e a degradação da substância. Tendo em vista esse fato, o dimetoato foi o agrotóxico selecionado para a execução do experimento de sorção.

\section{EFEITO DO PH E DA CONCENTRAÇÃO INICIAL DO DIMETOATO SOBRE A SORÇÃO}

Os resultados do experimento são mostrados na Tabela 3. Como as análises foram realizadas em duplicatas, esses resultados representam a média das duas determinações. Com base nos dados da tabela 3 é possível verificar que na maioria das amostras a quantidade do dimetoato sorvida diminui com o aumento do $\mathrm{pH}$. Comportamento contrário foi encontrado para as amostras coletadas nas profundidades de $0-0.30 \mathrm{~m}$ e $0.30-1.0 \mathrm{~m}$ que foram submetidas a uma concentração inicial de $20 \mathrm{mg} \cdot \mathrm{l}^{-1}$, nas quais a sorção decresce com a diminuição do $\mathrm{pH}$.

Como exemplo do comportamento predominante podem ser citadas as amostras do intervalo de 0-0.30 m (concentração inicial $10 \mathrm{mg}^{-1} \mathrm{l}^{-1}$. Para estas notou-se que em $\mathrm{pH} 5$ a quantidade sorvida foi de $0.024 \mathrm{mg} \cdot \mathrm{g}^{-1}$, enquanto que a $\mathrm{pH} 8$, a quantidade foi $0.017 \mathrm{mg} \cdot \mathrm{g}^{-1}$. Em porcentagem, estes valores representam 
24.1 e $16.6 \%$, respectivamente. Tal comportamento pode ser atribuído à variação dos valores de solubilidade do dimetoato com o $\mathrm{pH}$, a qual varia de $23.3 \mathrm{~g} . \mathrm{l}^{-1}$, em pH 5, a $25.0 \mathrm{~g} . \mathrm{l}^{-1}$, em $\mathrm{pH}$ 9. Geralmente, o aumento da solubilidade da molécula traduz a diminuição do seu caráter hidrofóbico, que contribui para a diminuição de sua sorção aos sedimentos (Rocha et al., 2002). Comportamento semelhante foi verificado por Rocha et al. (2002) ao estudar a retenção do herbicida imazaquin em solo tropical altamente intemperizado.

Verificando a tabela 3, nota-se que, de maneira geral, em concentração maior a sorção tende a ser menor independente do valor do $\mathrm{pH}$ do ensaio. As exceções são verificadas para as amostras do intervalo de 1.0-3.0 m, nas condições de $\mathrm{pH}$ igual a cinco, e para as amostras do intervalo $0.30-1.0 \mathrm{~m}$, nas condiçóes de $\mathrm{pH}$ igual a oito.

Ao estudar os efeitos dos componentes mineralógicos e orgânicos de alguns latossolos na adsorção do herbicida fluometuron, Benevenuto (1983) verificou que aumentando a concentração inicial da solução, aumentava também a quantidade sorvida pelos componentes do solo. Resultado semelhante era esperado para o presente estudo, já que o aumento da concentração inicial aumentaria a concentração de massa do soluto disponível para ser transferido da solução para a superfície dos sedimentos. No entanto, a situação contrária prevaleceu em $75 \%$ das amostras analisadas. Uma explicação possível para esse comportamento seria o fato das concentrações iniciais utilizadas no experimento terem sido superiores à capacidade de sorção dos sedimentos.

\section{EFEITO DOS TEORES DE MATÉRIA ORGÂNICA E ARGILA NA SORÇÃO}

Os teores de matéria orgânica das amostras estudadas e a quantidade de dimetoato sorvida por cada uma delas são mostrados na tabela 3. De acordo com os dados obtidos, verificase que para as amostras submetidas às condições de $\mathrm{pH} 5 \mathrm{e}$ concentração inicial de $10 \mathrm{mg} . \mathrm{l}^{-1}$, o teor de matéria orgânica influencia positivamente na quantidade sorvida, ou seja, quanto maior o teor de matéria orgânica maior é a quantidade sorvida. Para as amostras do intervalo de $0.0-0.30 \mathrm{~m}$, com $3 \%$ de matéria orgânica, a quantidade sorvida foi de $0.024 \mathrm{mg} \cdot \mathrm{g}^{-1}$, enquanto que para o intervalo de $0.30-1.0 \mathrm{~m}$, que contém $1.52 \%$ de matéria orgânica, a sorção foi de $0.019 \mathrm{mg} \cdot \mathrm{g}^{-1}$. Em condiçôes de $\mathrm{pH} 8$, para a profundidade $0.0-0.30 \mathrm{~m}$, com $3 \%$ de matéria orgânica, a quantidade sorvida foi de $0.017 \mathrm{mg} \cdot \mathrm{g}^{-1}$; quando o teor de matéria orgânica passou para $1.5 \%$, no nível inferior (0.30-1.0 m), a quantidade sorvida diminuiu para $0.008 \mathrm{mg} . \mathrm{g}$ 1. Comportamento semelhante é encontrado para as outras condições e principalmente para as amostras dos níveis mais superficiais $(0-1.0 \mathrm{~m})$.

Os resultados acima são semelhantes aos encontrados por Lopes et al. (2002) ao estudar a influência da matéria orgânica na adsorção do fungicida triadimenol pelo solo. Nesse estudo, os autores verificaram que a afinidade do triadimenol pela matriz aumenta com o teor de matéria orgânica.

A Tabela 3 apresenta o teor de argila e a quantidade sorvida pelos sedimentos das diversas profundidades. De acordo com essa tabela, as amostras submetidas à concentração inicial de 10 mg..$^{-1}$ e pH 5 apresentam aumento na quantidade sorvida quando aumenta o teor de argila. Nota-se que para as amostras do intervalo de $0.0-3.0 \mathrm{~m}$ a $1.0-3.0 \mathrm{~m}$ a quantidade sorvida aumentou de $0.024 \mathrm{mg} . \mathrm{g}^{-1}$ para $0.031 \mathrm{mg} . \mathrm{g}^{-1}$, enquanto o teor de argila passou de $4.84 \%$ para $6.25 \%$. Comportamento contrário é verificado para as amostras submetidas às condiçōes de concentração inicial de $20 \mathrm{mg}^{-1} \mathrm{l}^{-1} \mathrm{e} \mathrm{pH} \mathrm{8.} \mathrm{Nas} \mathrm{amostras} \mathrm{do}$ intervalo de $0.0-5.0 \mathrm{~m}$ a quantidade sorvida diminui de 0.024 mg. $\mathrm{g}^{-1}$ para $0.005 \mathrm{mg} \cdot \mathrm{g}^{-1}$ quando o teor de argila aumenta de $4.84 \%$ para $11.9 \%$. Comportamento similar éobservado também para as amostras dos intervalos mais superficiais, de 0.0 a $1.0 \mathrm{~m}$, nas diferentes condiçōes de $\mathrm{pH}$ e concentração inicial. Entretanto, essas variaçôes na quantidade de dimetoato sorvida não estão relacionadas com o teor de argila, uma vez que o dimetoato é relativamente estável em água quando o $\mathrm{pH}$ está na faixa de $2 \mathrm{a}$ 7. Assim, essa substância pode ser considerada como não-iônica para as condiçôes de $\mathrm{pH}$ utilizadas no experimento de sorção. Segundo Benevenuto (1983), para os compostos não-iônicos as cargas das argilas e sua superfície específica tornam-se sem muita importância para a sorção. Portanto, a variação da quantidade de dimetoato sorvida, observada no presente estudo, provavelmente não pode ser atribuída à variação do teor de argila e sim ao teor de matéria orgânica.

Constata-se, portanto, que o teor de matéria orgânica nos sedimentos é a propriedade mais importante na sorção do dimetoato. Seu comportamento não-iônico torna sem importância a variação do teor e do tipo da argila. Por outro lado, o caráter hidrofóbico do dimetoato aumenta a sua afinidade com a matéria orgânica.

\section{CONCLUSÕES}

Na bacia hidrográfica do igarapé Cumaru os agrotóxicos, entre eles o dimetoato, deltametrina, difenoconazole e mancozeb são utilizados com elevada freqüência, sem preocupação com a qualidade dos recursos hídricos.

A partir da análise de risco verificou-se que o dimetoato apresenta maior potencial para contaminar as águas subterrâneas, com índice GUS variando de 2,36 a 3,36. Mesmo o agrotóxico apresentando potencial de contaminação, esta pode não ocorrer desde que condições locais permitam a sorção e degradação da substância, daí a importância da realização dos experimentos de sorçāo.

O experimento mostrou que, em termos percentuais, a sorção do dimetoato pelos sedimentos da zona não-saturada variou de 


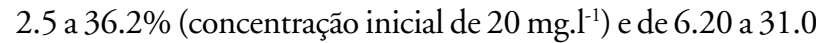
$\%$ (concentração inicial de $10 \mathrm{mg}^{\mathrm{l}^{-1}}$ ). Esses dados comprovam o elevado potencial de contaminação da água subterrânea por essa substância, devendo-se, principalmente, à sua mobilidade e baixa retenção.

A quantidade sorvida do agrotóxico avaliado se mostrou diretamente proporcional à quantidade de matéria orgânica presente nos sedimentos, sendo esse comportamento atribuído ao caráter hidrofóbico, tanto da matéria orgânica como do dimetoato.

Em relação ao teor de argila, notou-se que esse componente não influênciou na sorção do dimetoato, devido, principalmente, ao caráter não-iônico dessa substância. Tal característica faz com que a matéria orgânica seja o componente do solo mais importante na sorção do dimetoato.

$\mathrm{O} \mathrm{pH}$, por sua vez, exerce efeito contrário ao da matéria orgânica, ou seja, quanto mais elevado o seu valor, menor é a quantidade de dimetoato sorvida. Atribui-se esse efeito principalmente à diferença na solubilidade do dimetoato com a variação do $\mathrm{pH}$.

A quantidade de dimetoato sorvida diminuiu quando a concentração inicial foi elevada de 10 para $20 \mathrm{mg} \cdot \mathrm{l}^{-1}$. Esse resultado deveu-se provavelmente ao fato das concentrações iniciais utilizadas no experimento terem sido superiores à capacidade de sorção dos sedimentos.

Com base no presente estudo recomenda-se que seja efetuado o monitoramento das águas subterrâneas da bacia hidrográfica do igarapé Cumaru, a fim de verificar os níveis de ocorrência do dimetoato nessa águas, já que o contaminante mostrou sofrer pouca atenuação. Recomendam-se também estudos mais detalhados, a fim de conhecer o comportamento do dimetoato e de outros compostos que apresentem alto potencial de lixiviação. É igualmente desejável o uso de software que simule o movimento dos compostos químicos na zona não-saturada.

Embora o dimetoato tenha mostrado baixa retenção é necessário considerar que a alta evaporação da região e as temperaturas médias registradas poderão favorecer os processos de volatilização das moléculas. Além disso, deve-se considerar a influência da radiação solar da região que favorece a degradação dos agrotóxicos por fotólise.

\section{BIBLIOGRAFIA CITADA}

Amaral, C.M.L. 2001. Agricultura y Riesgo Ambiental en las Microcuencas del Cumaru y Caripi en la Amazonia Brasileña: effectos del uso de los agroquimicos. Dissertação de Mestrado, Turrialba, Centro Agronómico Tropical de Investigación y Enseñanza. 92pp.

Bastos, T.X; Pacheco, N.A. 1999. Características agrometeorológicas do município de Igarapé-Açu. In: seminário sobre manejo da vegetação secundária para a sustentabilidade da agricultura familiar na amazônia da oriental, Belém. Anais... Belém: EMBRAPA/ CNPq, p.51-58.

Benevenuto, J.G.F. 1983. Efeitos de componentes mineralógicos e orgânicos de alguns latossolos na Adsorção de Herbicida Fluometuron. Dissertação de Mestrado, Viçosa, Universidade Federal de Viçosa. 94pp.

The e-pesticide manual. 2001. 12 ed., version 2.1. The British Crop Protection Council.

Gao, J.P.; Maguhn, J.; Spitzauer, P.; Kettrup, A. 1998. Sorption of pesticides in the sediment of the teufelsweiher pond (Southern Germany) I: Equilibrium Assessments, efect of organic carbon content and pH. Water Res., 32 : 1662-1667.

Goés, A.M.; Truckenbrodt, W. 1980. Caracterização faciológica e interpretação ambiental dos sedimentos Barreiras na região Bragantina, nordeste do Pará. In: Congresso Brasileiro de Geologia, 31., Camboriú. Anais.... Camboriú: SBG, v 2, p. 766-771.

Goulart, A.T. 1982. Intemperismo químico de rochas graniticas na Zona Bragantina, nordeste do Pará. Belém. Dissertação de Mestrado, Universidade Federal do Pará. 47pp.

Gustafson, D.I. 1989. Groundwater ubiquity score: a simple method for assessing pesticide leachability. Environ. Toxicol. Chem., 8(4): 339-357.

Guimarães, G.A.; Bastos, J.B.; Lopes, E.C.1970. Métodos de análise fisica, química e instrumental de solos. Belém, Instituto de Pesquisas e Experimentação Agropecuária do Norte, Série: Química de solos. 112pp.

Kanashiro, M.; Denich, M. 1998. Possibilidades de utilização e manejo adequado de áreas alteradas e abandonadas na Amazônia Brasileira: estudos dos impactos Ambientais nas florestas e áreas abandonadas nos trópicos- SHIFT. Brasília: MCT/CNPq.

Leão, L.M. 2003. Caracterização hidrogeológica em área de agricultura intensiva na microbacia do Igarapé Cumaru, nordeste do Pará. Dissertação de mestrado, Universidade Federal do Pará. Belém, PA. 102pp.

Lopes, N.P.;Queiroz, M.E.L.; Neves, A.A. 2002. Influência da matéria orgânica na adsorção do fungicida triadimenol pelo solo. Química Nova, 25 (4) : 544-547.

Nemeth-Konda, L.; Füleky, GY.; Morovjan, GY.; Csokan, P. 2002. Sorption behaviour of acetochlor, atrazie, carbendazim, diazinon, imidacloprid and isoproturon on Hungarian Agricultural soil. Chemosphere, $48: 545-552$.

Pessoa, M.C.P.Y.; Gomes, M.A.F.; Neves, M.C.; Cerdeira, A.L.; Souza, M.D. 2003. Identificação de áreas de exposição ao risco de contaminação de águas subterrâneas pelos herbicidas atrazina, diuron e tebutiurn. Pesticidas: R. Ecotoxicologia e Meio Ambiente, 13: 111-122.

Poltroniéri, L.C. 1997. Agricultura, meio ambiente e saúde pública: A questão dos praguicidas no Brasil. Sociedade \& Natureza, 9 (17): 131-144.

Rocha, W.S.D.; Alleoni, L.R.F.; Regitano, J.B.; Tornisiello, V.L. 2002. Sorption of Imazaquin in soils with positive balance of charges. Chemosfhere, 49: 263-270. 


\section{ACTA}

Silva, A.B. 1994. Evolução química das águas subterrâneas. Águas subterrâneas, 7.

Singh, N. 2002. Sorption behaviour of triazole fungicides in indian soils and its correlation with soil properties. J. Agric. Food. Chem., $50: 6434-6439$.

Spark, K. M.; Swift, R.S. 2002. Effect of soil composition and dissolved organic matter on pesticide sorption. The Science of the Total Environment, 298 (1):147-161.

Suguio, K. Introdução à Sedimentologia. 1973. São Paulo, SP: Ed. da Universidade de São Paulo.
Vieira, E.M.; Prado, A.G.S.; Landgraf, M.D.; Rezende, M.O.O. 1999. Estudo da adsorção/desorção do ácido 2,4 diclorofenoxiacético $(2,4 \mathrm{D})$ em solo na ausência e presença de matéria orgânica. Quimica Nova, 22: 305-308.

Wickel, B. 2004. Water and nutrient dynamics of a humid tropical agricultural watershed in Eastern Amazônia. Zetrun für Entwicklungsforschung. Center for development Reseach. University of Born. Ecology and Development Series. n.21.

Recebido em 18/11/2004

Aceito em 27/02/2007 
